\title{
Journal of Membrane Science \& Technology
}

\section{Membrane Oscillation and Heisenberg Uncertainties}

\section{Kalmbach HE Gudrun*}

Mathematic Informatics Naturewissenchaften, Technic, Germany

"Corresponding author: Kalmbach HE Gudrun, Mathematics Professor, Inventor of MINT (Mathematic Informatics Naturewissenchaften, Technic), Germany, Tel: +49(0)3025418-499; E-mail: mint-01@maxi-dsl.de

Received date: July 20, 2018; Accepted date: August 7, 2018; Published date: August 14, 2018

Copyright: (c) 2018 Gudrun KHE. This is an open-access article distributed under the terms of the Creative Commons Attribution License, which permits unrestricted use, distribution, and reproduction in any medium, provided the original author and source are credited.

\section{Abstract}

In a new model for deuteron states a superposition of three colour charge whirls from the strong interaction SI for deuteron is introduced. It is called an rgb-graviton whirl which generates a nucleons location as a dynamical time crystal. Its SI action as membrane oscillation is described in this article (membrane oscillation, half cones). Added is for the weak interaction WI in the second part of the article that its action for the time crystal of deuteron is different with an explanation for the Heisenberg uncertainties $\mathrm{HU}$ in hedgehog caps and deuteron isospin exchange.
\end{abstract}

Keywords Colour charge; Deuteron; Heisenberg uncertainty; Membrane; Nucleon

\section{Introduction}

The new model for deuteron states is developed for extending the known symmetry $\mathrm{U}(1) \times \mathrm{SU}(2) \times \mathrm{SU}(3)$ of the standard model of physics through the Moebius transformations such that gravity GR and the Einstein relativities are added (Figure 1). It requires that beside this new symmetry on a deuteron bounding Riemannian sphere the measuring apparatus for quantum mechanics is expanded by the use of Gleason operator measures. In a geometrical description they are generated by orthogonal coordinate or vector triples like the spin coordinates for space vector rotor (Figure 2). The spin length is replaced by numerical values for other energies like the length of a momentum vector, a mass or angular momentum. The model is described in an octonian vector space. Its 8 dimensions are then projected down to suitable subspaces. WI and electromagnetism is described in the physics space time coordinate space $x y z t, t$ time, $x, y, z$ space coordinates. For six postulated energies the distribution of projected vectors for the $\mathrm{HU}$ is shown as the six paired colour charge caps in the hedgehog figure for deuterons (Figures 3 and 4). Spherical SI coordinates a new GR interpretation is that the WI and SI coordinates for deuteron are set separately in octonians ans are in special relativistic motion against one another (Figures WI, SI top views). The GR action of rgb-gravitons is described in the first part of the article (Figure 5). There are 5 videos which demonstrate the different dynamics inside a deuteron (Figure 6).

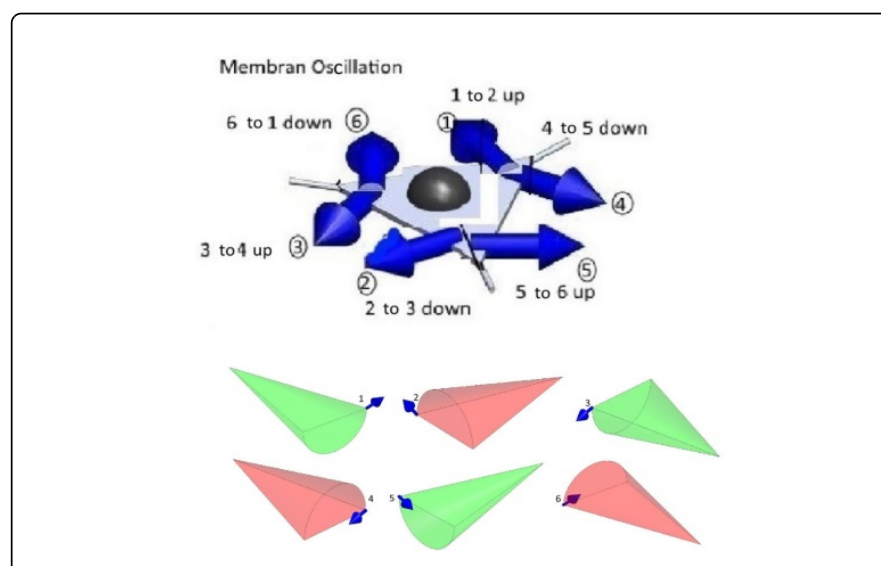

Figure 1: Rotation of the momentum vector blue, six red green half cone membranes.

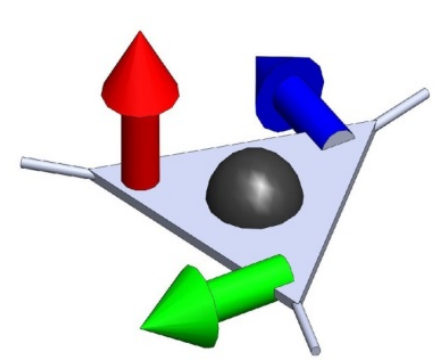

Figure 2: Vector rotor in 6 cyclic positions. 


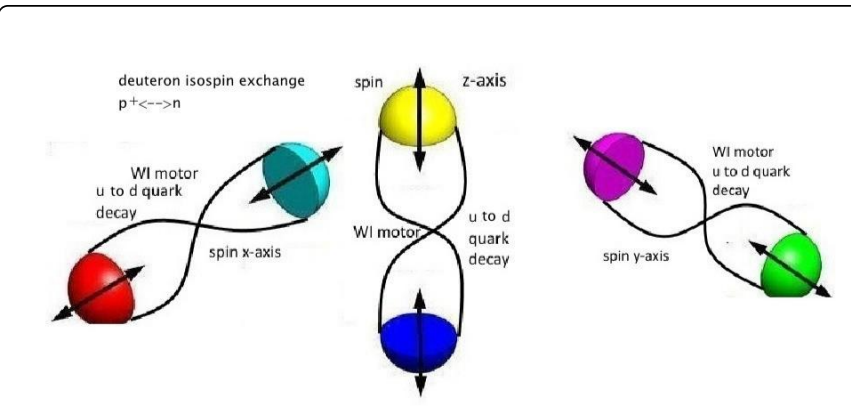

Figure 3: $\mathrm{W}+$ decay rotor for a $\mathrm{u}$ - to a d-quark, shown between paired polar caps energy on the $\mathrm{x}-, \mathrm{z}$ - and $\mathrm{y}$-axis.

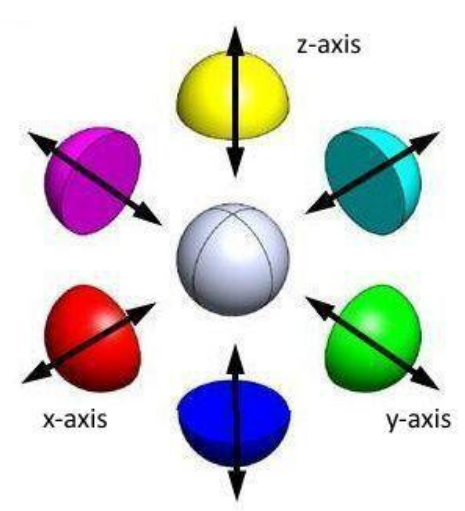

Figure 4: hedgehog caps covering a deuteron atomic kernel for energy transfers.

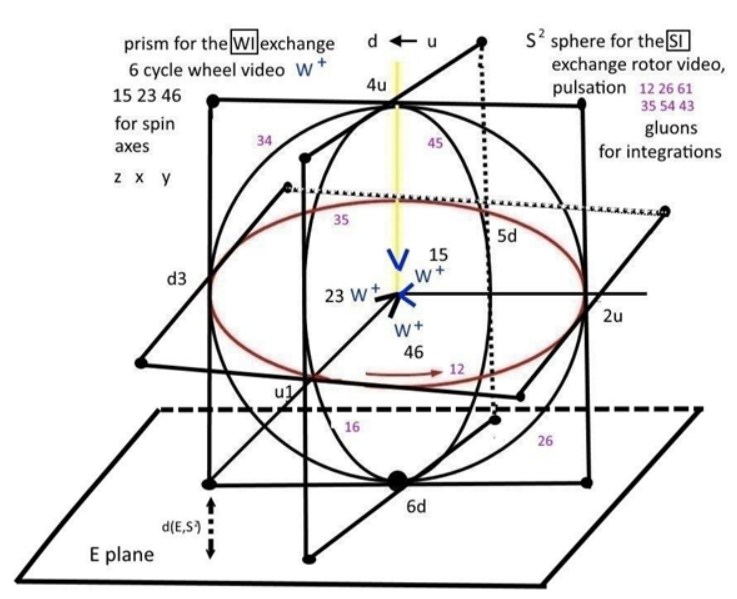

Figure 5: Prism for deuteron as time crystal.
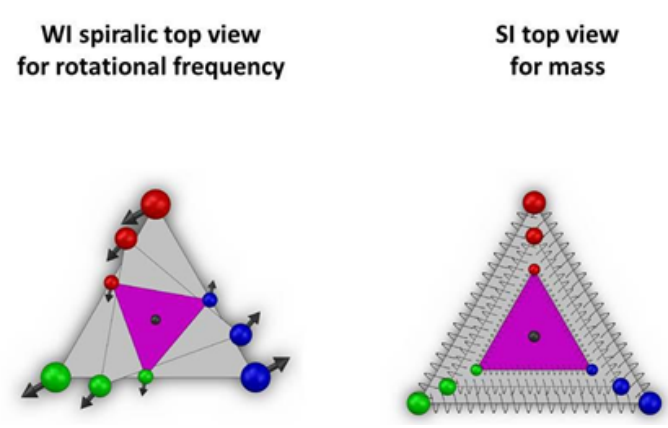

Figure 6: Nucleon 3 Bohr radii contraction and expansion.

\section{Results and Discussion}

\section{Membranes}

In the MINT Wigris model [1] of the author the neutral colour charge of a nucleon was named a gravity GR field quantum rgbgraviton whirl as superposition of three colour charge whirls red, green, blue (Figure 1). In nucleons inner dynamics the rgb-graviton is important; its dynamics is coming from the triangle symmetry D3 of a quark triangle. Quarks have a periodically fixed location through a linear momentum vector which rotates with D3. Fixing the vertices for a nucleon quark triangle is a time cycle, generating a time crystal. At the rotation in Figure 1, there are two more vectors attached (generating the six green, red membrane half cones) as a cross product triple like the three spin coordinates in space or as in the triple for angular momentum (Figure 2) rotor. This is a vector version of the rgbgraviton. In the D3 rotation the other two vectors set barycentrical coordinates for the nucleon, fixing in the middle of the figure a barycenter. The whirl motion up-down is a presentation of the D3 symmetry not used in physics and oscillates cyclic in $1234561 \ldots . . n$ the quark triangle area to a half cone up or down.

\section{Heisenberg uncertainty pairings}

In the mathematical deuteron states rotor dynamics of MINTWigris associated the three Heisenberg uncertainties $\mathrm{HU}$ with a weak WI boson $\mathrm{W}+$ exchange between paired $\mathrm{u}$-, $\mathrm{d}$-quarks in a deuteron (Figure 3) prism along the signed $\mathrm{x}-$, $\mathrm{y}$ - or $\mathrm{z}$-axis (second list of three figure W+ decay) (Figure 4) in the inner deuteron space [1]. The polar caps in the hedgehog figure show the $\mathrm{HU}$ in form of (position as radius $r$ on $+x$ electrical energy EM(pot), with momentum $p=m v$ ( $m$ mass, $v$ speed) on $-x$, mass energy $E($ pot)), (time on $+z$, magnetic energy $\mathrm{E}$ (magn), energy $\mathrm{E}$ as frequency $\mathrm{E}=\mathrm{hf}$, $\mathrm{h}$ the Planck constant, $\mathrm{f}$ inverted time interval $\Delta \mathrm{t}$ on $-\mathrm{z}$, kinetic energy $\mathrm{E}($ kin)), (angle on $+\mathrm{y}$, heat energy $\mathrm{E}$ (heat), angular momentum on $-\mathrm{y}$ ), rotation energy $\mathrm{E}(\mathrm{rot})$. The interpretation for this pairing is explained by the mathematical inversion at a circle. This inversion uses the first square Pauli matrix $\sigma 1$ ( $a i i=0$, aij=1 for $I \neq j, i, j=1,2$ ). Scalings are not mentioned. The HU coupling is for the $\mathrm{x}$-axis: beween radius $[\mathrm{r}, 1]$ and potential (replace momentum with speed $\mathrm{v}$ by a differential $\mathrm{d} / \mathrm{dr})((1 / \mathrm{r}), 1)$ as projective vectors; $z$-axis: beween a time interval $[\Delta t, 1]$ and frequency (replace frequency by a time difference in $\mathrm{d} / \mathrm{dt})[(1 / \Delta \mathrm{t}), 1]$ as projective vectors ; $\mathrm{y}$-axis: between heat $\mathrm{E}$ (heat) transfer $\Delta \mathrm{T}$ as angle $\varphi$ measured interval $[(\mathrm{d} \varphi=\Delta \mathrm{T}), 1]$ and angular momentum $\mathrm{L}=\operatorname{rxp}$ (replace $\mathrm{L}$ by a differential $\omega)[\omega=(\mathrm{d} \varphi / \mathrm{dt}), 1]$ as angular speed in $\mathrm{L}=\mathrm{J} \omega$. 
For scalings, in the first case, as radius of inversion the Schwarzschild radius $r=R s$ can be taken, for the second and last case a radius 1 . The third case is postulated newly in the MINT- Wigris model. Heat transfer is not in physics associated by a matrix inversion with an angular speed (momentum). But this arises naturally when the former two inversions are applied to this case.

In the first and second $\mathrm{HU}$ case the marix $\sigma 1$ matrix can be from right multiplied with the vector. In the last case, the symmetry $\mathrm{D}^{3}$ of a quark triangle in a deuterons nucleon (proton or neutron) has to be used [1]. Heat has a coefficient matrix of the (angle) cross ratio $\mathrm{z} /(1-\mathrm{z})$, applying (projectively) $\sigma 1$ from the left, the coefficient matrix of angular momentum (1-z)/z is obtained (also reversely from $\omega$ to $d \varphi$ ).

In case experiments can be designed, the heat generated angular momentum L (or reversely: from the L energy generated heat) is expected by these matrices multiplications. Quoted from [2] in the development of the early universe physics and my model claim that first quarks from a 5- dimensional Schmutzer field are generated. This is called creation and lets open why the postulated fermionic series are created. Three energies are quoted before: time, length, temperature. In the time development of the universe it is postulated that from this Schmutzer field time crystals as nucleons and hadrons arise.

In [1] the energy bifurcation of the Schmutzer 5- dimensional sphere, an electrical charge or field separates from a gravitational or mass field. Two polar quarks are generated after assuming a big bang, having mass and an electrical charge. Then quotation from [2] the colour charges postulated for the SI with the space $S^{3} \mathrm{x} \mathrm{S}^{5}$ as a trivial fibre bundle blow up from $S$ have an rgb-colour charge whirl generated $\mathrm{S}^{3}$ as blow up of $\mathrm{S}$ for generating nucleons. Projected like Euclidean spin coordinate vectors they are pointing in the $\mathrm{x}, \mathrm{y}$ and $-\mathrm{z}$ coordinate direction and generate with their endpoints in a nucleon bounding sphere $S^{2}$ a quark triangle. For the nucleon as time crystal they are rotating as a triple using the quark triangle symmetry for their changes in a time cycle of order 6 . This way barycentrical coordinates arise in the quark triangle which determine the nucleons barycenter. The difference to EM/WI is that in the symmetry used the product of two triangle reflections generate its flat rotation by 120 degree angle while the EM/WI Pauli matrix symmetry uses the real cross product for their two reflection matrices which is then also a reflection. After 1 second in the early universes development it is assumed that neutrinos can escape from nucleons a atomic kernels can be generated from them with respect to the upper three HU inversions at a loop. Astronomy can be quoted: when two spiralic rotating huge galaxies rotate about a common barycenter, they hit and their energy is distributed newly. Assume for a big bang that this applies for the universe: two extremely huge energy systems P,Q did hit. Then the three HU inversions took place: the extreme high rotational speed was inverted to the big heat at the Planck time after the inversion, Planck length as $\mathrm{r}$ or $\Delta \mathrm{x}$ was inverted from the GR potential as scaled $1 / \mathrm{r}$ or $1 / \Delta \mathrm{x}$ and time $\Delta \mathrm{t}$ was inverted from the P,Q frequency $1 / \Delta t[3,4]$.

In the Figure 3 prism a plane $\mathrm{E}$ is shown. As new symmtry in [1] the Moebius transformations MT on a nucleon or deuteron bounding hedgehog caps Riemannian sphere of $S^{2}$ are added to the standard models symmetry $\mathrm{U}(1) \times \mathrm{SU}(2) \times \mathrm{SU}(3)$ of physics. In a central projection of $S^{2}$ onto $E$ the rgb-graviton action can be used in MT matrix form to change the distance $\mathrm{d}\left(\mathrm{E}, \mathrm{S}^{2}\right)$. It shows then the projected nucleon triangle perspective in three Bohr radii sizes, small, middle large, as a GR length contraction/expansion. The WI coordinates have a spiralic rotation added the strong SI interactions coordinates show a barycentrical contraction/expansion. Generated heat can be used for the expansion.

\section{Conclusion}

The new model can be used to replace in quantum mechanics the old infinite dimensional Hilbert space for the case of deuteron states to the 8-dimensional octonian vector space. New findings are the description of an inner space time and geometry for a deuteron atomic kernel as a time crystal. The dynamics is discrete, bound to field quantum exchanges. In addition the Heisenberg uncertainties get an explanation in Figure 5, a pairing of energy and coordinates through the indicated inversions. Central projections used for gravity actions in Figure 6 are also new. Experimental data are today in July 2018 not available, but several videos which show the possible membrane/ graviton, special relativistic coordinate actions for deutron and how the HU pairings occur in the hedgehog description of deuteron. The mathematical base comes from the new symmetry of Moebius transformations, using projective geometries in the 8 octonian dimensions and the measuring Gleason operators from [1]. For them, described by scaled coordinate vector or energy triples, experimental data are needed, similar to the Stern-Gerlach experiment for spin.

\section{References}

1. Gudrun Kalmbach HE (2017) Mint Wigris. SF J Nuclear Science 2: 1-4.

2. Gudrun KHE (2017) MINT-Wigris, Scholar's Press, Beau Bassin; Contents in the internet by Deutsche Nationalbibliothek Frankfurt/M, Germany 1.

3. Gudrun Kalmbach HE (2017) Deuteron States. Journal of Physics 1: 1-17.

4. Poston T, Stewart I (1978) Catastrophe Theory and its Applications (Pitman, London, UK). 